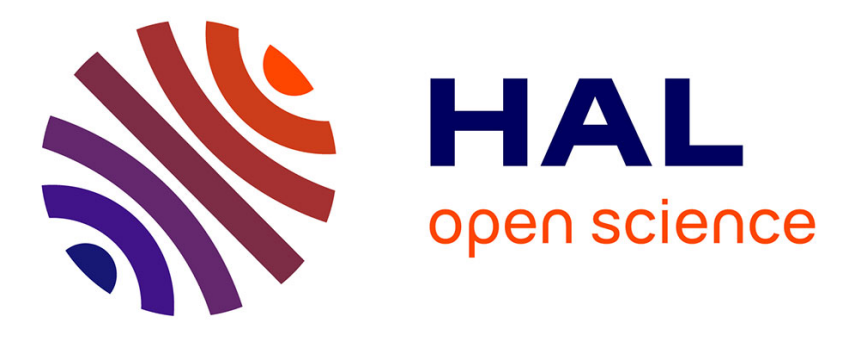

\title{
Multispectral Demosaicing using Intensity-based Spectral Correlation
}

Sofiane Mihoubi, Olivier Losson, Benjamin Mathon, Ludovic Macaire

\section{To cite this version:}

Sofiane Mihoubi, Olivier Losson, Benjamin Mathon, Ludovic Macaire. Multispectral Demosaicing using Intensity-based Spectral Correlation. 5th IEEE International Conference on Image Processing Theory, Tools and Applications (IPTA 2015), Nov 2015, Orléans, France. pp.461-466, 10.1109/IPTA.2015.7367188 . hal-01196983

\section{HAL Id: hal-01196983 \\ https://hal.science/hal-01196983}

Submitted on 10 Sep 2015

HAL is a multi-disciplinary open access archive for the deposit and dissemination of scientific research documents, whether they are published or not. The documents may come from teaching and research institutions in France or abroad, or from public or private research centers.
L'archive ouverte pluridisciplinaire HAL, est destinée au dépôt et à la diffusion de documents scientifiques de niveau recherche, publiés ou non, émanant des établissements d'enseignement et de recherche français ou étrangers, des laboratoires publics ou privés. 


\title{
Multispectral Demosaicing using Intensity-based Spectral Correlation
}

\author{
Sofiane Mihoubi ${ }^{1}$, Olivier Losson ${ }^{1}$, Benjamin Mathon ${ }^{1}$ and Ludovic Macaire ${ }^{1}$ \\ ${ }^{1}$ CRIStAL - UMR 8219 \\ Université de Lille - Sciences et Technologies, Bâtiment P2 \\ 59655 Villeneuve d'Ascq Cedex France \\ email: sofiane.mihoubi@etudiant.univ-lille1.fr, olivier.losson@univ-lille1.fr, \\ benjamin.mathon@univ-lille1.fr, ludovic.macaire@univ-lille1.fr
}




\begin{abstract}
Single-sensor color cameras, which classically use a color filter array (CFA) to sample RGB channels, have recently been extended to the multispectral domain. To sample more than three wavelength bands, such systems use a multispectral filter array (MSFA) that provides a raw image in which a single channel level is available at each pixel. A demosaicing procedure is then needed to estimate a multispectral image with full spectral resolution. In this paper, we propose a new demosaicing method that takes spectral and spatial correlations into account by estimating the level for each channel. Experimental results show that it provides estimated images of better quality than classical methods.
\end{abstract}

\title{
Index Terms
}

Multispectral image demosaicing, Multispectral filter array, Spectral correlation, Spatial correlation.

\section{INTRODUCTION}

Digital color cameras are limited in their ability to faithfully capture colors because their are usually limited to three wavelength bands of the visible electromagnetic spectrum. To overcome this limitation, multispectral imaging has recently emerged to provide more spectral resolution, with applications in various fields such as medical imaging [1], military surveillance [2], satellite imaging [3, 4], or vision inspection for food quality control [5].

Multispectral images can be captured in full definition by selecting a different optic filter for each spectral channel. Papers [5] and [2] give a good review of the available technologies (among which tunable filters) that provide such images. They are available for nearly twenty years but another technology emerges that captures a raw image with a multispectral filter array (MSFA) [6]. Each pixel in this raw image is defined by the level of a single channel according to the MSFA pattern, while the other channels have to be estimated to recover each channel in full definition. This process known as demosaicing is similar in its principle to the estimation of RGB components in singlesensor color cameras fitted with a Bayer color filter array (CFA). But whereas CFA demosaicing is a well-studied problem for more than thirty years [7], MSFA demosaicing is a recent subject with new issues.

This paper is organized as follows. First we present the considered MSFA, the demosaicing problem, and the related works about multispectral demosaicing. Second, we review the existing methods that can be used with the retained MSFA. We then propose a new method that is presented in section 4 and experimentally evaluated in section 5 . 


\section{MSFA DEMOSAICING}

\subsection{Multispectral filter arrays}

To ensure manufacturing practicability and demosaicing feasibility, MSFAs are always defined by a basic repetitive pattern. Two important criteria must also be considered in the MSFA design [8]: spectral consistency, which requires that pixels should always have the same number of neighbors of a certain channel, and spatial uniformity, which requires that the MSFA samples each channel as evenly as possible. Figure 1 illustrates two MSFAs that meet these criteria: the "Uniform" MSFA proposed by Aggarwal and Majumdar [9] and a $4 \times 4$ MSFA where each of the $K=16$ channels appears only once in the basic pattern. Few other MSFAs have been proposed in the literature [6] but most of them are not compliant with the above design criteria and/or exhibit a dominant spectral channel (that appears more than the others), which does not allow for a high spectral resolution.

In the following, we only consider the 16-channel MSFA shown in Fig. 1(b) for two main reasons. First, sampling all channels only once in the basic pattern provides the most compact MSFAs, which allows us to consider a high number of channels $(K=16$ whereas authors of [9] only test up to $K=6$ ) with limited estimation errors (as shown in the experimental section). Second, we motivate the choice of a square non-redundant pattern to mimic IMEC's snapshot multispectral camera [10], that is—-to our knowledge — the sole off-the-shelf MSFA-based system available on the market today.

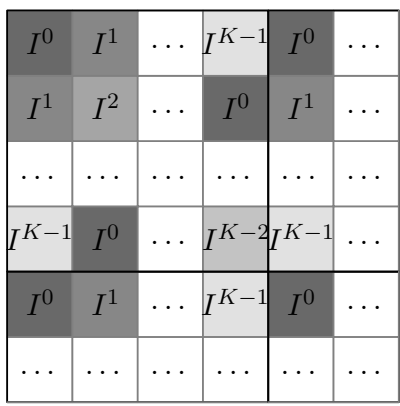

(a) Uniform pattern [9]

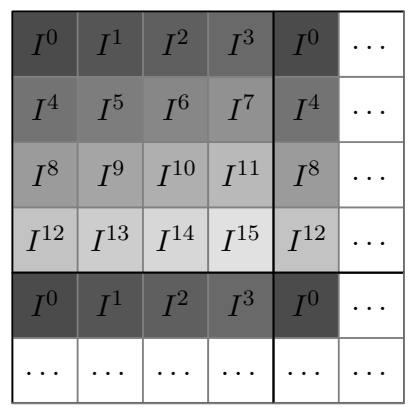

(b) $\sqrt{K} \times \sqrt{K}$ pattern, $K=16$

Fig. 1. Examples of $K$-channel MSFAs with two different patterns. $I^{k}$ means that only the level of the $k$-th channel is available at that pixel.

\subsection{Multispectral demosaicing}

A single-sensor multispectral camera fitted with an MSFA provides a raw image $I^{M S F A}$ with $X \times Y$ pixels. Each pixel $P(x, y)$ of $I^{M S F A}$, whose level is $I_{x, y}^{M S F A}, x \in\{0,1, \ldots, X-1\}, y \in$ $\{0,1, \ldots, Y-1\}$, is associated with a single channel $k \in\{0,1, \ldots, K-1\}$. Let us consider the 
image I made of the fully-defined $K$ channel planes $I^{k}$. This image is called the reference image because it is often used as a reference to evaluate the demosaicing quality even if it is unavailable in practice. Then, $I^{M S F A}$ can be seen as a spectrally-sampled version of $\mathbf{I}$ according to the spatial coordinates $(x, y)$ :

$$
I_{x, y}^{M S F A}=I_{x, y}^{k}, \text { with } k=x \bmod \sqrt{K}+\sqrt{K} \cdot(y \bmod \sqrt{K}) .
$$

At each pixel of the raw image $I^{M S F A}$, only one out of the $K$ channels is available and the levels of the $K-1$ others are missing. Demosaicing $I^{M S F A}$ is to obtain an estimated image $\hat{\mathbf{I}}$ with $K$ fully-defined channels among which $K-1$ ones are estimated at each pixel:

$$
\hat{\mathbf{I}}_{x, y}=\left(\hat{I}_{x, y}^{0}, \ldots, \hat{I}_{x, y}^{k-1}, I_{x, y}^{k}, \hat{I}_{x, y}^{k+1}, \ldots, \hat{I}_{x, y}^{K-1}\right),
$$

where $k$ is given by Eq. (1).

\subsection{Related works}

In recent years, a few multispectral demosaicing algorithms have been proposed. The first outstanding work is provided by Miao et al. [8] who build a generic binary tree to generate MSFA patterns, and who perform demosaicing by a binary tree-based edge-sensing (BTES) method. This algorithm is dedicated to MSFA patterns with a dominant channel, which is not optimal for square patterns. Monno et al. [11-13] propose three demosaicing algorithms for a specific 5-channel MSFA pattern that contains a dominant green channel associated to $50 \%$ of known values in the raw image. The original algorithm [11] uses an adaptive kernel upsampling, which is improved in [12] by using a guided filter, and further on in a third algorithm [13] that integrates a residual interpolation. Wang et al. extend the DWT-based [14] and the median filtering-based demosaicing algorithms [15] from the RGB color space to the multispectral domain, but the first approach also relies on a dominant green channel to estimate the luminance while the second one provides a poor demosaicing result [16]. Aggarwal and Majumdar [9] take the spatial (inter-pixel) correlation into account by learning the interpolation weights from a database. However, the performance of this approach highly depends on the learning database.

Brauers and Aach [17] take the spectral (inter-channel) correlation into account in a demosaicing algorithm based on the spectral channel difference planes. Mizutani et al. [16] propose to iterate Brauers and Aach's estimation a number of times given by the spectral proximity of the considered pair of spectral channels. Brauers and Aach's algorithm is proposed for a $3 \times 2$ MSFA but can easily be adapted to other patterns. 


\section{ADAPTED METHODS}

In this section, we present three adaptations of the previous methods to square non-redundant MSFAs. These are referred to as the Weighted Bilinear (WB) method, Spectral Difference (SD) method and Iterated Spectral Difference (ISD) method. They are adapted from the $3 \times 2$ MSFA to the $4 \times 4$ MSFA below for clarity sake, without loss of generality.

\subsection{Weighted Bilinear method (WB)}

The weighted bilinear interpolation of Brauers and Aach [17] works in two steps:

- First, use a binary mask to obtain the sparse raw image $\tilde{I}^{k}$ for each channel $k, k \in\{0,1, \ldots, 15\}$. This image contains non-zero levels only at the locations where real measurement data exist. The binary mask for each spectral channel $k$ is defined at each pixel $(x, y)$ as:

$$
m^{k}(x, y)= \begin{cases}1 & \text { if } k=x \quad \bmod 4+4 \cdot(y \bmod 4) \\ 0 & \text { otherwise }\end{cases}
$$

The sparse raw image is then defined as:

$$
\tilde{I}^{k}=I^{M S F A} \odot m^{k},
$$

where $\odot$ denotes the element-wise product.

- Second, compute each estimated channel $\hat{I}^{k}$ as:

$$
\hat{I}^{k}=\tilde{I}^{k} * H,
$$

where $*$ is the convolution operator, and $H$ is a low-pass filter defined as:

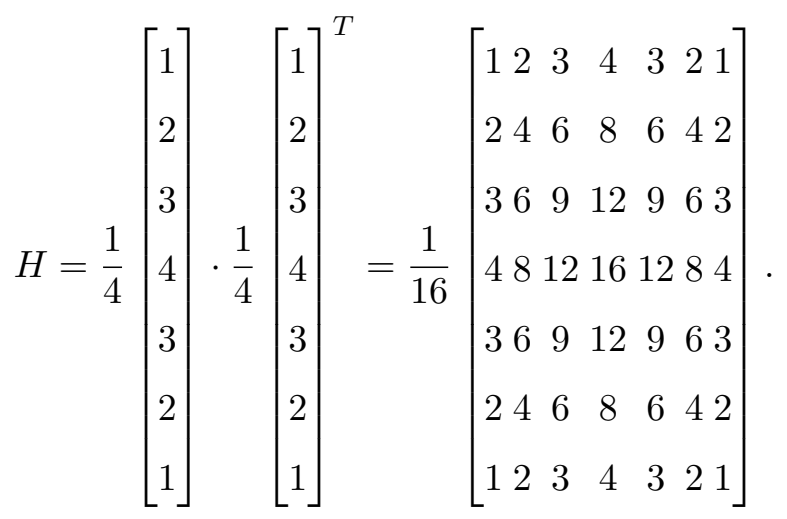

The weight of each neighbor represented by the convolution filter of Eq. (6) decreases with respect to its spatial distance to the central pixel.

Such interpolation is considered as the most intuitive method for MSFA demosaicing and gives reference results for the other methods. 


\subsection{Spectral Difference method (SD)}

Brauers and Aach [17] also propose a method that relies on the above WB interpolation but takes spectral correlation into account. We extend it here to any non-redundant $4 \times 4$ MSFA. This method starts by computing a fully-defined multispectral image $\hat{\mathbf{I}}_{W B}=\left\{\hat{I}_{W B}^{k}\right\}_{k=0}^{15}$ by WB interpolation (see Eq. (5)). The next steps are:

1) First, for each ordered pair $(k, l)$ of spectral channels, compute the sparse channel difference given by:

$$
\Delta^{k l}=\hat{I}_{W B}^{k} \odot m^{l}-\tilde{I}^{l} .
$$

where $\tilde{I}^{l}$ is defined by Eq. (4). Thus, the sparse channel difference $\Delta^{k l}$ is only non-zero at the locations where $m^{l}(x, y)=1$.

2) Second, estimate a fully-defined channel difference $\hat{\Delta}^{k l}$ by WB interpolation. For this purpose, convolve each sparse channel difference $\Delta^{k l}$ with the filter $H$ (see Eq. (6)) to estimate this difference at each pixel:

$$
\hat{\Delta}^{k l}=\Delta^{k l} * H
$$

3) Third, for each channel $l \in\{0,1, \ldots, 15\}$, compute the estimation $\hat{I}^{l}$ at pixels where $m^{k}(x, y)=$ 1 as:

$$
\hat{I}^{l}=\sum_{k=0}^{15}\left(\tilde{I}^{k}-\hat{\Delta}^{k l}\right) \odot m^{k} .
$$

The 16 channels $\hat{I}^{l}, l=0 . .15$, are fully-defined and form the demosaiced spectral image $\hat{\mathbf{I}}$.

\subsection{Iterative Spectral Difference method (ISD)}

Mizutani et al. [16] further take the correlation between two channels into account, that is strong when the central wavelengths of these channels are close. They propose to improve Brauers and Aach's method by updating the channel difference at each iteration if channels $k$ and $l$ are spectrally close. The closer are the wavelengths, the more iterations are needed. The number of iterations $N^{k l}$ is given by:

$$
N^{k l}=\exp \left(-\frac{\left|\lambda_{l}-\lambda_{k}\right|-100}{20 \sigma}\right)
$$

where $\lambda_{l}-\lambda_{k}$ denotes the difference between the central wavelengths of channels $k$ and $l$, and $\sigma$ is a parameter that is set to 1.74 by the authors. Equation (10) implies that the number of iterations is set to 1 if $\left|\lambda_{l}-\lambda_{k}\right|>100 \mathrm{~nm}$, and the parameter value $\sigma=1.74$ implies $N^{k l}=10$ iterations when the difference between the wavelengths equals $20 \mathrm{~nm}$.

The algorithm starts by estimating all sparse channel differences $\Delta_{0}^{k l}$ (see Eq. (7)) and all channels $\hat{I}_{0}^{l}$ (see Eq. (9)), $k, l=0 . .15$. The three steps for each iteration $t>0$ are: 
1) First, compute the updated sparse channel difference $\Delta_{t}^{k l}$ as:

$$
\Delta_{t}^{k l}= \begin{cases}\hat{I}_{t-1}^{k} \odot m^{l}-\tilde{I}^{l} & \text { if } t \leq N^{k l} \\ \Delta_{t-1}^{k l} & \text { otherwise. }\end{cases}
$$

2) Second, estimate a fully-defined channel difference $\hat{\Delta}_{t}^{k l}$ by WB interpolation. For this purpose, convolve each channel difference $\Delta_{t}^{k l}$ with the filter $H$ (see Eq. (6)) to estimate this difference at each pixel:

$$
\hat{\Delta}_{t}^{k l}=\Delta_{t}^{k l} * H
$$

3) Third, estimate each channel $\hat{I}_{t}^{l}, l=0 . .15$, as:

$$
\hat{I}_{t}^{l}=\sum_{k=0}^{15}\left(\tilde{I}^{k}-\hat{\Delta}_{t}^{k l}\right) \odot m^{k} .
$$

\section{INTENSITY-DIFFERENCE DEMOSAICING}

In the previous methods, the correlations between channels whose wavelengths are too distant (like 400 and $700 \mathrm{~nm}$ ) are taken into account, which is prone to generate demosaicing errors. Our proposal is to consider the intensity for demosaicing, assuming that the correlation between each channel and the intensity is higher on average than the correlation between channels considered pairwise.

\subsection{Intensity interpolation}

We define the intensity level at each pixel as the average level over all the channels. Since only one channel is available at each pixel in the raw image $I^{M S F A}$, we assume that the intensity is strongly correlated between neighboring pixels. Taking this spatial correlation into account, we directly estimate the intensity on the raw image by applying a smoothing filter $M$.

The size of $M$ is that of the smallest odd-size window that includes each channel at least once in the raw image. Each element of $M$ is set to $\frac{1}{j}$, where $j$ is the number of times when the channel associated to the neighbor occurs in the support window of $M$. This filter is normalized afterwards so that all its elements sum up to 1 .

Consider for instance the $4 \times 4$ MSFA of Fig. 1(b). Then, the size of $M$ is $5 \times 5$ and centering $M$ at pixel $(2,2)$ yields four available levels for channel 0 , two levels for channels $1-3,4,8$ and 12 , and a single level for the other channels. Considering any other central pixel in $I^{M S F A}$ provides the 
same filter $M$ for such a $4 \times 4$ MSFA, namely:

$$
M=\frac{1}{64} \cdot\left[\begin{array}{lllll}
1 & 2 & 2 & 2 & 1 \\
2 & 4 & 4 & 4 & 2 \\
2 & 4 & 4 & 4 & 2 \\
2 & 4 & 4 & 4 & 2 \\
1 & 2 & 2 & 2 & 1
\end{array}\right] .
$$

The intensity image $I^{M}$ is then defined as:

$$
I^{M}=I^{M S F A} * M
$$

We propose two methods for demosaicing raw images by using the intensity image: Intensity Difference (ID) method and Iterative Intensity Difference (IID) method.

\subsection{Intensity Difference (ID)}

The algorithm is divided into four successive steps:

1) First, compute the intensity image $I^{M}$ (see Eq. (15)).

2) Second, for each channel $k \in\{0,1, \ldots, 15\}$, compute the sparse difference $\Delta^{k}$ between the level of channel $k$ and the intensity at the locations where channel $k$ is available in $I^{M S F A}$ :

$$
\Delta^{k}=\tilde{I}^{k}-I^{M} \odot m^{k} .
$$

3) Third, for each channel $k \in\{0,1, \cdots, 15\}$, estimate the fully-defined difference by WB interpolation (see Eqs. (5) and (6)):

$$
\hat{\Delta}^{k}=\Delta^{k} * H
$$

4) Finally, estimate each channel $k$ by adding the intensity and the difference:

$$
\hat{I}^{k}=I^{M}+\hat{\Delta}^{k} .
$$

Equation (18) is based on the assumption that the channel $k$ is strongly correlated with the intensity image.

The ID algorithm combines the spatial and spectral correlations to estimate the missing levels. We assume that the intensity between neighboring pixels is correlated. This spatial correlation also justifies the interpolation of the difference between raw channels and the intensity. Once this difference has been estimated, we assume that each spectral channel is strongly correlated with the intensity at each pixel to estimate the fully-defined channel. 


\subsection{Iterative Intensity Difference (IID)}

We improve the estimation by iterating the algorithm ID as follows.

We first estimate an initial fully-defined multispectral image $\hat{\mathbf{I}}_{0}=\left\{\hat{I}_{0}^{k}\right\}_{k=0}^{15}$ by using the ID algorithm. The three steps for each iteration $t>0$ are then:

1) First, compute the new intensity image $I_{t}^{M}$ as:

$$
I_{t}^{M}=\frac{1}{16} \cdot \sum_{k=0}^{15} \hat{I}_{t-1}^{k} .
$$

Note that the intensity is here estimated as the average level over all the channels whereas it is estimated by WB interpolation in ID.

2) Second, compute the new fully-defined difference (see Eqs. (16) and (17)) as:

$$
\hat{\Delta}_{t}^{k}=\left(\tilde{I}^{k}-I_{t}^{M} \odot m^{k}\right) * H,
$$

3) Third, estimate each channel as:

$$
\hat{I}_{t}^{k}=I_{t}^{M}+\hat{\Delta}_{t}^{k}
$$

These steps are iterated a number of times $N$ to provide the estimated multispectral image $\hat{\mathbf{I}}=$ $\left\{\hat{I}_{N}^{k}\right\}_{k=0}^{15}$.

\section{EXPERIMENTAL RESULTS}

\subsection{Comparison of the different methods}

We compare the performances reached by our proposed demosaicing methods (ID and IID) with those provided by Weighted Bilinear (WB), Spectral Difference (SD) and Iterative Spectral Difference (ISD) methods. The number $N$ of iterations required by our IID method has been set to 7 since it provides the best results (see section 5.2.1). Our experiments are performed on 32 multispectral images with 16 channels from the CAVE database [18]. These images have been rendered with CIE D65 illuminant. The wavelength center of each channel ranges from 400 to $700 \mathrm{~nm}$ with a step of $20 \mathrm{~nm}$. Each image is spectrally sampled with the MSFA pattern proposed in Fig.1 $(b)$ to simulate a raw multispectral image. The quality of an estimated image is evaluated by computing the average Peak Signal-to-Noise Ratio (PSNR) between the estimated and reference channels. The PSNR values provided by the different methods on the 32 images are shown in Table 1 where the highest PSNR value for each image is displayed in bold face. It shows that the quality of the images reconstructed by our proposed methods outperforms the quality of images obtained by the other presented methods on 31 images. Our iterative method improves the average PSNR by more than $1 d B$ w.r.t. existing methods and by $0.6 d B$ w.r.t. its non-iterative version. 
We have selected the "Fake and Real Lemon" and "Flowers" CAVE images to visually compare the results of each method. The reference and reconstructed images are converted from the 16-channel domain to the sRGB color space for illustration purposes. This transformation is performed using the CIE XYZ $2^{\circ}$ standard observer functions and we use the reference white D65 to convert values from $\mathrm{XYZ}$ to the sRGB color space. Figures 2 and 3 show that the image demosaiced by the WB method is strongly blurred. The SD and ISD methods generate severe zipper effect and color artifacts. These artifacts are fairly reduced with the ID method, that also generates less blur than WB. IID provides sharper edges but a bit more visual color artifacts than ID.

\subsection{Discussion}

It is interesting to generalize our methods to other sizes of square MSFA patterns than $4 \times 4$. For this purpose, we consider $2 \times 2$ and $3 \times 3$ MSFAs ranging from 400 to $700 \mathrm{~nm}$ with respective steps of 100 and $40 \mathrm{~nm}$. Our ID and IID methods can be directly applied to these MSFAs, provided that the filter $M$ is adapted. For a $2 \times 2$ and a $3 \times 3$ MSFA pattern, the filter $M$ is respectively defined as:

$$
M=\frac{1}{16} \cdot\left[\begin{array}{lll}
1 & 2 & 1 \\
2 & 4 & 2 \\
1 & 2 & 1
\end{array}\right] \text { and } M=\frac{1}{9} \cdot\left[\begin{array}{lll}
1 & 1 & 1 \\
1 & 1 & 1 \\
1 & 1 & 1
\end{array}\right]
$$

We focus our experimental discussion on the quality of demosaicing with respect to the number of iterations and on the comparison of the PSNR values between the methods applied to different MSFAs.

5.2.1. Number of iterations: We compare the results obtained by the proposed IID method, $N$ ranging from 0 to 15 iterations in order to determine the best number of iterations. The results are shown in Fig. 4 for the three considered MSFAs. The $X$-Axis represents the number of iterations $N$, and the $Y$-Axis represents the average PSNR over the 32 images. As it can be seen, iterating the estimation 7 times increases the PSNR by $0.5 d B$ for the $4 \times 4$ MSFA. The best PSNR is obtained when the number of iterations is 4 for the $3 \times 3$ MSFA, and 0 for the $2 \times 2$ MSFA. This last result can be explained by the impact of the spectral correlation during the iteration process and by the high distance between the wavelengths of the four channels.

5.2.2. Methods applied to the three MSFAs: Figure 5 shows the average PSNR on the 32 CAVE images obtained by the different methods. For the $2 \times 2$ MSFA, the WB method provides the best PSNR because of the weak spectral correlation. The ID method gives similar results because it mainly uses spatial correlation. For the $3 \times 3$ MSFA, the SD and ISD methods give results which are similar to the WB method and our ID and IID methods increase the PSNR by $1 d B$. We can conclude from 


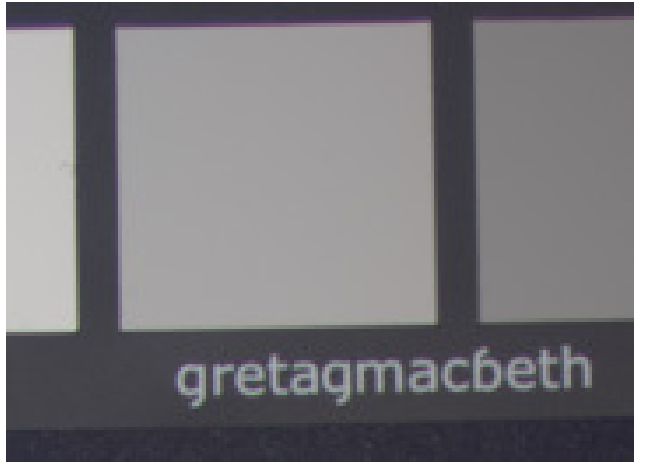

(a) Reference image

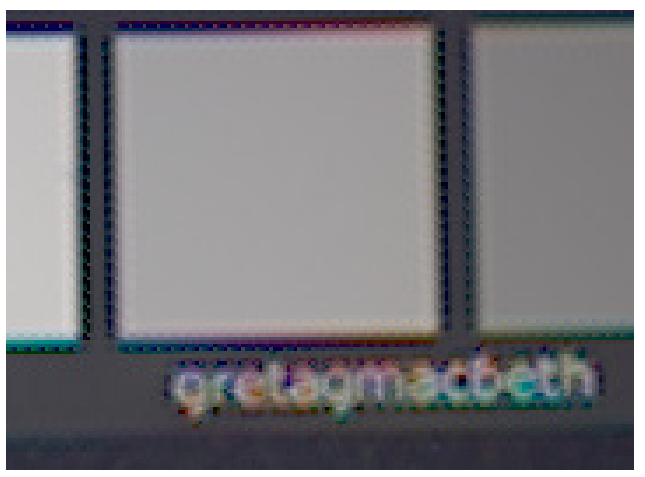

(c) SD method

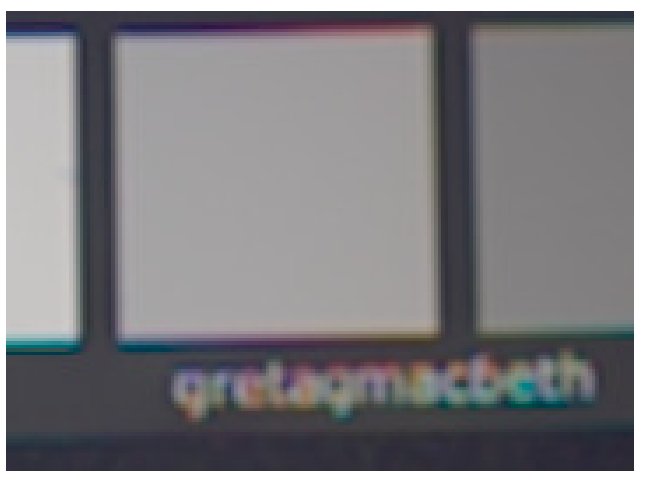

(e) ID method

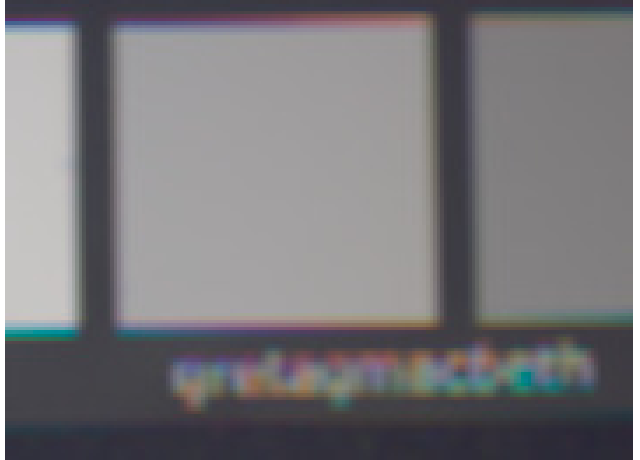

(b) WB method

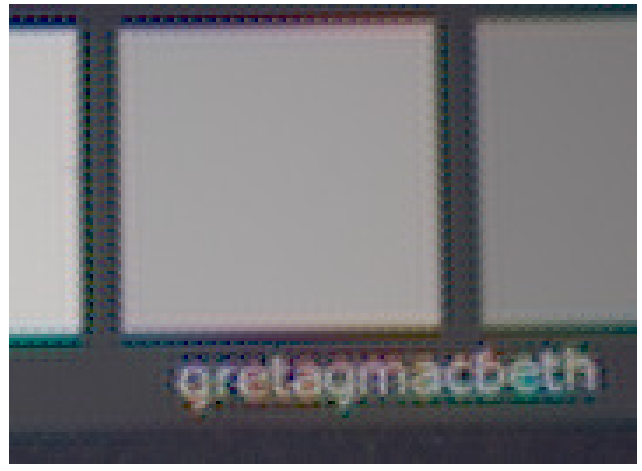

(d) ISD method

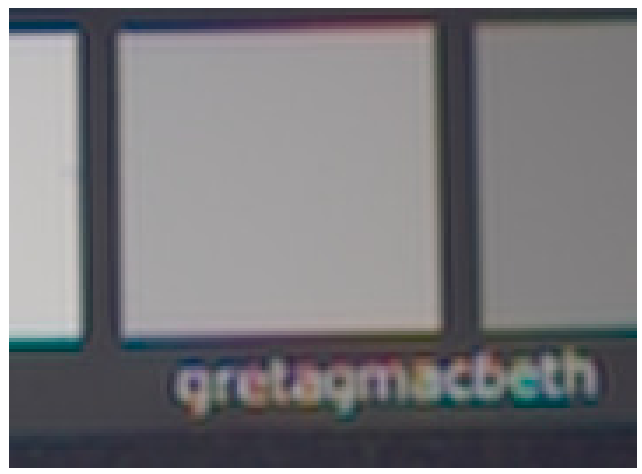

(f) IID method

Fig. 2. sRGB renderings for each method on a central area of the "Fake and real Lemons" CAVE image.

these experimental results that our demosaicing methods based on the intensity outperform classical approaches when the size of the pattern is large, i.e., when the number of channels is higher than 4 .

\section{CONCLUSION}

In this paper, we have shown that the assumption about spectral correlation between channels should be carefully used since it is not fully verified when the spectral bands are distant. That leads us to propose a new demosaicing method based on the correlation between each channel and 


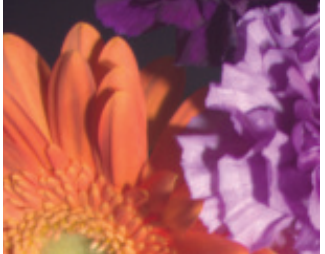

(a) Reference image

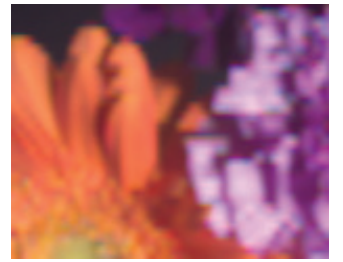

(b) WB method

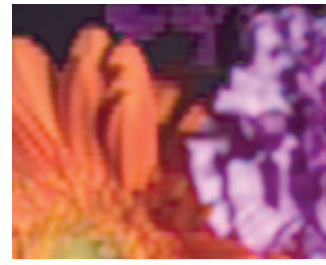

(e) ID method

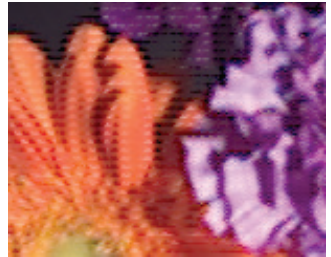

(c) SD method

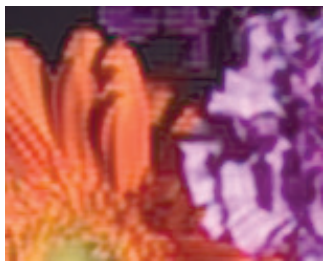

(f) IID method

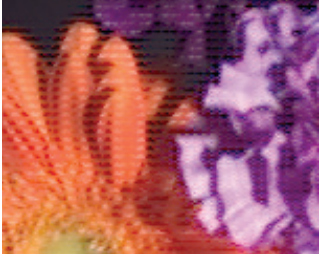

(d) ISD method

Fig. 3. sRGB renderings for each method on a central area of the "Flowers" CAVE image.

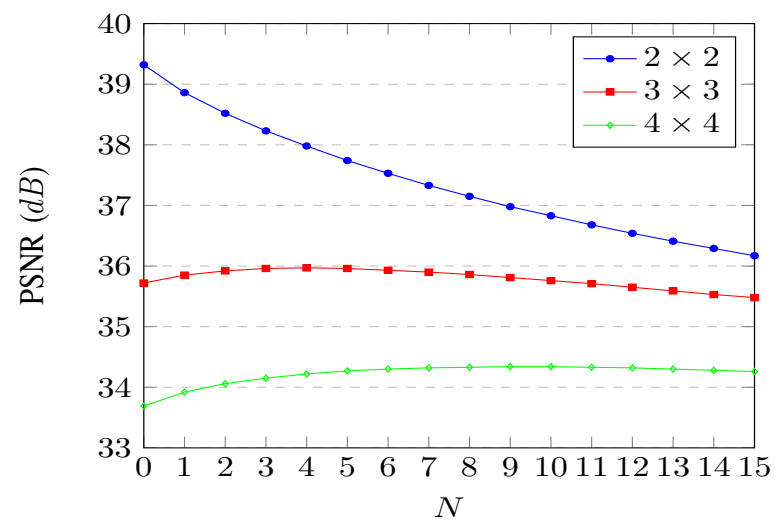

Fig. 4. PSNR (average values over the 32 CAVE images) provided by the IID method with respect to the number of iterations $N$ for the $2 \times 2,3 \times 3$, and $4 \times 4$ MSFAs.

the intensity. Two versions of the method have been presented and experimental results illustrate the improvement brought by iterating the estimation. Extensive experiments show that the proposed algorithms outperform the other ones in terms of PSNR. The new interpolation method improves the estimated image quality and approximately retains the sharpness of the reference one while reducing color artifacts and zipper effect. Future works will focus on reducing the spatial shift between the different estimated channels in order to remove color artifacts.

\section{ACKNOWLEDGMENT}

This work has been performed thanks to the ANR-11-EQPX-23 IrDIVE platform. 


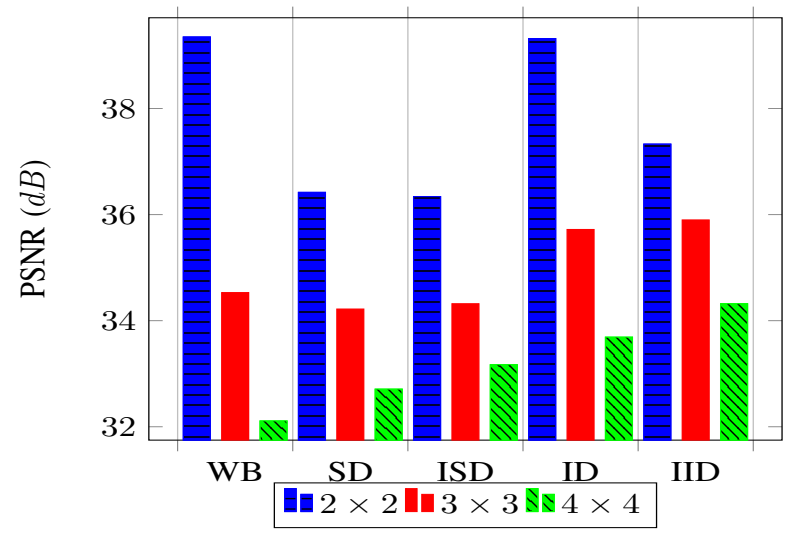

Fig. 5. PSNR (average values over the 32 CAVE images) provided by the 5 methods for the $2 \times 2,3 \times 3$, and $4 \times 4$ MSFAs.

\section{REFERENCES}

[1] G. Lu and B. Fei. Medical hyperspectral imaging: A review. J. of Biomedical Optics, 19(1):010901, Jan. 2014.

[2] F. Vagni. Survey of hyperspectral and multispectral imaging technologies. Technical report, Research and Technology Organisation of the NATO, May 2007.

[3] C. Yang, J. H. Everitt, Q. Du, B. Luo and J. Chanussot. Using high-resolution airborne and satellite imagery to assess crop growth and yield variability for precision agriculture. Proceedings of the IEEE, 101(3):582-592, Mar. 2013.

[4] H. Z.M. Shafri, E. Taherzadeh, S. Mansor and R. Ashurov. Hyperspectral remote sensing of urban areas: An overview of techniques and applications. Research J. of Applied Sciences, Engineering and Technology, 4(11):1557-1565, 2012.

[5] J. Qin, K. Chao, M. S. Kim, R. Lu and T. F. Burks. Hyperspectral and multispectral imaging for evaluating food safety and quality. J. of Food Engineering, 118(2):157-171, 2013.

[6] P.-J. Lapray, X. Wang, J.-B. Thomas and P. Gouton. Multispectral filter arrays: Recent advances and practical implementation. Sensors, 14(11):21626-21659, Nov. 2014.

[7] X. Li, B. K. Gunturk and L. Zhang. Image demosaicing: A systematic survey. In Procs. SPIE Conf. on Visual Communications and Image Processing (VCIP'08), volume 6822, pages 68221J1-15, San Jose, California, USA, Jan. 2008.

[8] L. Miao, H. Qi, R. Ramanath and W.E. Snyder. Binary tree-based generic demosaicking algorithm for multispectral filter arrays. IEEE Trans. on Image Processing, 15(11):3550-3558, Nov. 2006.

[9] H. K. Aggarwal and A. Majumdar. Single-sensor multi-spectral image demosaicing algorithm using learned interpolation weights. In Procs. of the 2014 Int. Geoscience and Remote Sensing Symp. (IGARSS 2014), pages 2011-2014, Québec City, Québec, Canada, Jul. 2014.

[10] B. Geelen, N. Tack and A. Lambrechts. A compact snapshot multispectral imager with a monolithically integrated per-pixel filter mosaic. In Procs. of the SPIE 8974, Advanced Fabrication Technologies for Micro/Nano Optics and Photonics VII, volume 8974, pages 89740L-89740L-8, San Francisco, California, USA, Feb. 2014.

[11] Y. Monno, M. Tanaka and M. Okutomi. Multispectral demosaicking using adaptive kernel upsampling. In Procs. of the 18th IEEE Int. Conf. on Image Processing (ICIP 2011), pages 3157-3160, Brussels, Belgium, Sep. 2011.

[12] Y. Monno, M. Tanaka and M. Okutomi. Multispectral demosaicking using guided filter. In Procs. of the SPIE Electronic Imaging Annual Symp. (SPIE'12): Digital Photography VII, volume 8299, pages 829900-82990O-7, Burlingame, California, USA, Jan. 2012. 
[13] Y. Monno, D. Kiku, S. Kikuchi, M. Tanaka and M. Okutomi. Multispectral demosaicking with novel guide image generation and residual interpolation. In Procs. of the IEEE Int. Conf. on Image Processing (ICIP 2014), pages 645-649, Paris, France, Oct. 2014.

[14] X. Wang, J.-B. Thomas, J. Y. Hardeberg and P. Gouton. Discrete wavelet transform based multispectral filter array demosaicking. In Procs. of the 2013 Colour and Visual Computing Symp. (CVCS 2013), pages 1-6, Gjøvik, Norway, Sep. 2013.

[15] X. Wang, J.-B. Thomas, J. Y. Hardeberg and P. Gouton. Median filtering in multispectral filter array demosaicking. In Procs. of the SPIE Electronic Imaging Annual Symp. (SPIE'13): Digital Photography IX, volume 8660, pages 86600E-86600E-10, Burlingame, California, USA, Feb. 2013.

[16] J. Mizutani, S. Ogawa, K. Shinoda, M. Hasegawa and S. Kato. Multispectral demosaicking algorithm based on interchannel correlation. In Procs. of the 2014 IEEE Visual Communications and Image Processing Conf., pages 474-477, Valletta, Dec. 2014.

[17] J. Brauers and T. Aach. A color filter array based multispectral camera. Procs. 12. Workshop Farbbildverarbeitung, Ilmenau, Germany, Oct. 2006.

[18] F. Yasuma, T. Mitsunaga, D. Iso and S. K. Nayar. Generalized assorted pixel camera: Postcapture control of resolution, dynamic range, and spectrum. IEEE Trans. on Image Processing, 19(9):2241-2253, Sep. 2010. 
Table 1. PSNR $(d B)$ of CAVE images estimated by the different methods. FaR stands for "Fake and Real" and RaF for "Real and Fake".

\begin{tabular}{|c|c|c|c|c|c|}
\hline Image & WB & SD & ISD & ID & IID \\
\hline Balloons & 38.77 & 39.54 & 39.81 & 40.49 & 41.19 \\
\hline Beads & 25.04 & 24.91 & 24.51 & 26.15 & 26.21 \\
\hline $\mathrm{CD}$ & 35.72 & 34.57 & 33.42 & 36.21 & 35.88 \\
\hline $\begin{array}{l}\text { Chart and stuffed } \\
\text { toy }\end{array}$ & 27.83 & 28.43 & 29.19 & 28.92 & 29.84 \\
\hline Clay & 34.82 & 33.32 & 32.98 & 35.78 & 35.79 \\
\hline Cloth & 22.94 & 24.42 & 25.26 & 24.35 & 25.18 \\
\hline Egyptian statue & 35.93 & 37.45 & 38.22 & 37.54 & 38.53 \\
\hline Face & 34.45 & 35.25 & 36.01 & 35.92 & 36.62 \\
\hline FaR Beers & 35.94 & 36.30 & 36.69 & 37.63 & 37.70 \\
\hline FaR food & 35.33 & 35.40 & 35.60 & 36.73 & 36.75 \\
\hline FaR Lemon slices & 32.15 & 32.96 & 33.65 & 33.41 & 33.94 \\
\hline FaR Lemons & 35.66 & 36.39 & 37.32 & 37.26 & 38.40 \\
\hline FaR Peppers & 35.10 & 35.69 & 36.24 & 37.79 & 38.34 \\
\hline FaR Strawberries & 33.29 & 34.73 & 35.58 & 35.01 & 36.20 \\
\hline FaR Sushi & 34.43 & 34.95 & 35.49 & 36.21 & 36.57 \\
\hline FaR Tomatoes & 32.43 & 33.37 & 34.19 & 33.71 & 34.40 \\
\hline Feathers & 29.41 & 30.56 & 31.16 & 31.23 & 32.47 \\
\hline Flowers & 31.65 & 32.12 & 32.30 & 33.58 & 33.93 \\
\hline Glass tiles & 25.42 & 26.21 & 26.76 & 26.81 & 27.86 \\
\hline hairs & 32.24 & 33.77 & 34.77 & 33.80 & 34.87 \\
\hline Jelly beans & 26.64 & 28.36 & 29.24 & 28.66 & 29.82 \\
\hline Oil painting & 28.52 & 28.70 & 29.38 & 28.54 & 29.57 \\
\hline Paints & 28.02 & 28.03 & 28.64 & 30.70 & 30.87 \\
\hline Photo and face & 34.01 & 35.39 & 36.03 & 36.19 & 37.53 \\
\hline Pompoms & 35.76 & 35.22 & 34.87 & 36.36 & 35.93 \\
\hline RaF Apples & 34.92 & 35.25 & 36.02 & 36.96 & 37.68 \\
\hline RaF Peppers & 33.06 & 33.84 & 34.76 & 34.71 & 35.91 \\
\hline Sponges & 32.21 & 31.60 & 31.66 & 33.41 & 33.25 \\
\hline Stuffed toys & 33.47 & 34.01 & 33.78 & 36.02 & 35.96 \\
\hline Superballs & 36.04 & 36.85 & 36.95 & 37.70 & 38.39 \\
\hline Thread spools & 30.04 & 31.42 & 32.54 & 32.00 & 33.60 \\
\hline Watercolors & 26.36 & 27.69 & 28.38 & 28.33 & 29.17 \\
\hline AVERAGE & 32.11 & 32.71 & 33.17 & 33.69 & 34.32 \\
\hline
\end{tabular}

